\title{
Areas del cerebro que participan en el proceso de lectura literal en sordos señantes: un caso de estudio
}

\author{
Brain areas associated with the literal reading \\ process in deaf user of sing language: a case study
}

\section{Adriana Rojas G. Fonoaudióloga \\ Mg Desarrollo educativo y social Investigadora Fundación Universitaria María Caro}

\section{RESUMEN}

El proceso de enseñanza o aprendizaje de la lectura como segunda lengua para la población sorda señante plantea varias interrogantes que buscan dar respuesta al bajo desempeño observado en el desarrollo de dicha actividad cognitiva. En esta situación se reconocen factores psicológicos, neurocognitivos y contextuales de los cuales depende el éxito del proceso. Objetivo: La presente investigación busca describir las zonas del cerebro que se activan cuando un usuario de una lengua ágrafa realiza una lectura literal en su segunda lengua. Método: Se plantea un enfoque cualitativo, de tipo descriptivo, a través de un estudio de caso único. Para la recolección de la información se emplea una electroencefalografía cuantitativamapeo cerebral. Resultados: El comportamiento del registro durante toda la aplicación presentó una variación significativa que coincide con el lóbulo temporal, específicamente en las áreas de asociación auditiva. Conclusiones: Al no existir huellas mnémicas con información fonológica, no se establece una relación grafema-fonema, es decir, no se evidencia una transformación de la palabra escrita en la representación fonológica del sonido de la misma como vía para acceder al léxico mental. Estos datos fonológicos se han remplazado por una información viso espacial que se alimenta directamente de la percepción visual, o bien, indirectamente, por medio de la generación de imágenes visuales.

Palabras claves: lectura, sordo, aprendizaje, enseñanza, segunda lengua, cerebro.

\begin{abstract}
The teaching or learning process of reading in a second language for deaf population, who are users of sign languages, pose several questions concerning the poor performance which they have in this cognitive activity. In this activity, there are recognized psychological, neurocognitive and environmental factors, which are vital for a successful process. Objetive: The aim of this research study is to describe the areas of the brain activated when a user of a language without a writing system performs a literal reading in their second language. Method: For the present research study, it was adopted a qualitative approach, with a descriptive type, through a single case study, using for the collection of information a quantitative electroencephalography or cerebral mapping. Results: The behaviour of the record throughout the application presented a remarkable change which matches with the temporal lobe, areas of hearing association. Conclusions: In the absence of memory traces with phonological information, there is not a grapheme-phoneme relationship, that is to say, there is no evidence of a transformation of the written word in the phonological representation of it as a way to access the mental lexicon. These phonological data have been replaced by optical-space information which is directly fed from visual perception or indirectly fed from the generation of visual imaging.
\end{abstract}

Key words: reading, deaf, learning, teaching, second language, brain. 


\section{Introducción}

Una mirada rápida al panorama terapéutico y educativo de los sordos permite ver que, tradicionalmente en Colombia, las propuestas para atender a dicha población han dado respuesta de manera tangencial a sus necesidades de formación y, en particular, al aprendizaje de la lectoescritura, situación que se evidencia en los bajos niveles de desempeño alcanzados en esta área ${ }^{1}$.

Siendo los sordos señantes personas bilingües, necesitan emplear varias modalidades de comunicación para ser efectivas en el cumplimiento de los distintos roles inherentes a cada etapa del ciclo vital. La lectura se constituye, pues, en una herramienta que no solo es empleada para acceder al conocimiento, sino también para comunicarse con las personas de su entorno ${ }^{1}$.

Históricamente la lectura y la escritura han sido enseñadas a los estudiantes sordos a través de metodologías que son réplicas de la enseñanza para los oyentes. Al no obtener resultados positivos en dicho proceso de enseñanza-aprendizaje se han generado diversos análisis alrededor de este ${ }^{2}$, los que además responden a la evolución del concepto de persona sorda desde una visión médico-clínica hacia una socioantropológica. Esta última perspectiva reconoce a la lectura y a la escritura como una modalidad de comunicación y como segunda lengua para el sordo señante, . Esta segunda lengua es aprendida luego de haber adquirido la de señas como primera lengua y se caracteriza por ser una de carácter viso gestual y ágrafa ${ }^{3}$.
La lectura y la escritura se constituyen en una herramienta de poder inventada por el ser humano que le sirve para comunicarse, para organizar sus pensamientos, como memoria externa, para extender y promover la comprensión de motivos y sentimientos de otros individuos y culturas y para crear formas nuevas de llevar a cabo otras acciones humanas ${ }^{4}$.

Así mismo, es un instrumento social que permite el desarrollo individual. En efecto, saber leer y escribir otorga poder y cambia la participación de los sujetos en el mundo. Por ello, la lectura es vista como un proceso de naturaleza comunicativa y cognoscitiva y se constituye en una valiosa herramienta para comunicarse con otros y acceder al conocimiento $^{1,20}$.

El presente estudio de caso asume la lectura como un proceso complejo en el que las funciones viso espaciales están unidas con funciones oculomotrices, lo que hace necesaria la participación de las áreas de asociación y de áreas motoras frontales para llevar a cabo dicha actividad. El acto de la lectura está asociado con la organización compleja de varias zonas del cerebro que se interrelacionan, por lo que su análisis permite conocer el proceso cerebral implicado.

De igual modo, la lectura es abordada desde un proceso léxico que depende de un almacenamiento preexistente en algún tipo de léxico mental. De acuerdo con la experiencia de la práctica profesional, en la mayoría de los casos, el niño sordo se acerca a la lectura con un bagaje de conocimientos y habilidades considerablemente particular debido a la diferencia lingüística. Por otra parte, el hecho de 
que la seña dependa del hemisferio izquierdo, pese a su organización espacial, indica que hay una representación de espacio "lingüístico» en el cerebro completamente distinta del espacio «topográfico» ordinario $^{5}$. Esto quiere decir que la seña utiliza algunas vías neuronales necesarias para el habla gramatical y otras generalmente relacionadas con procesos visuales.

En relación con los mecanismos neurobiológicos de la lectura descritos para la población oyente, autores como Ortiz ${ }^{6}$ afirman que la corteza visual tiene como función recibir e interpretar los estímulos visuales y comunicarse con otras áreas.

Esta doble función implica dos áreas neurofuncionales específicas diferenciadas: el área visual primaria, determinada por el área estriada 17 (áreas occipitales primarias) y el área visual secundaria para las áreas periestriada y paraestriada 18 y 19, respectivamente, que tienen una función relacionada con el proceso lector, dada su conexión con el área terciaria parietal 39 de Brodmann y la terciaria temporal, correspondiente al área 37 de Brodmann. La integración e interrelación de estas áreas con las de Broca y Wernicke permite acceder a la interpretación de los espacios dentro de la escritura, así como también a la orientación espacial, ambos aspectos relevantes en el proceso lector para la identificación de cada grafema, desde sus rasgos particulares, hasta la forma global del mismo. La interrelación entre estas áreas permite la interpretación global del texto al posibilitar la identificación de diferentes grafemas, sílabas, palabras y frases. Por último, la asociación con áreas fronto temporales permite el reconocimiento semántico de los símbolos gráficos. La anterior descripción corresponde a las áreas específicas e inespecíficas corticales de la lectura para oyentes sin diagnósticos asociados.

Por otra parte, investigaciones relacionadas con el presente estudio reportan la relación de zonas del cerebro con el aprendizaje de segundas lenguas (lengua auditivo verbal y su representación viso espacial) en la población oyente, como es el caso de Newman-Norlund, R.D., Frey, S.H., Petitto, L.A. and Grafton, S.T. ${ }^{18}$ y Perani, et al. ${ }^{25}$.

Así mismo Bellugi ${ }^{5}$ estudió la estructura de la lengua de señas y su sustrato neuronal en relación con diversas lesiones cerebrales. Neville, H., Bavelier, D., Corina, D., Rauschecker, J., Karni, A., Lalwani, A., Braun, A., Clark, V., Jezzard, P. and Turner, R ${ }^{26}$ incluyó en su estudio a personas sordas y oyentes en quienes observó, a través de imágenes de resonancia magnética, observó la organización cerebral durante el proceso lector en inglés y la lengua de señas americana. Investigaciones más recientes hacen referencia, en su mayoría, al rol que tiene el procesamiento fonológico en el aprendizaje de la lectura en la población sorda ${ }^{27}$ y el aprendizaje de la lectura y la escritura en relación con la adquisición tardía de una lengua.

Teniendo en cuenta los antecedentes investigativos y la condición lingüística particular de la población sorda se plantea, entonces, un estudio que describa la ruta cerebral en el proceso lector en personas sordas señantes, partiendo desde los procesos perceptivos, pasando por el reconocimiento 
de la palabra, su contraste con el léxico mental -que para el caso particular se ha estructurado a partir de una lengua ágrafa - para llegar, finalmente, a la construcción de una estructura semántica que integra con sus propios conocimientos. Esta información puede posibilitar, a futuro, el replanteamiento de las metodologías y estrategias empleadas por los maestros y fonoaudiólogos para promover el uso de la lectura en la población sorda, así como también prevenir problemas en su aprendizaje, o bien, ser más asertivos en la selección de estrategias compensatorias aplicables en los casos que evidencien una dificultad diagnosticada para el aprendizaje de esta modalidad comunicativa.

Por esta razón, el objetivo de la presente investigación es describir las zonas del cerebro que se activan cuando un usuario de una lengua ágrafa realiza una lectura literal en su segunda lengua, aspecto que de acuerdo con lo descrito en párrafos anteriores, no ha sido estudiado.

\section{Método}

La investigación se planteó desde un enfoque cualitativo con un método descriptivo y se diseñó como un ejercicio no experimental y transversal de campo. Para la selección de la unidad de análisis se estableció contacto con una institución educativa de la ciudad de Medellín, Colombia, que alberga a estudiantes sordos señantes en educación básica, secundaria y media y cuya filosofía responde a los principios de la enseñanza bilingüe bicultural ${ }^{21}$.

En un primer momento, se solicitó a los profesores seleccionar a aquellos estudiantes de su grupo que no usaran ayuda auditiva (implante coclear o audífono) y que tuvieran un nivel de comprensión lector literal (información básica que todo director de grupo conoce).

De un total de 20 estudiantes se seleccionaron 13 menores. Posteriormente, durante una conversación telefónica con cada represente legal, se recogió información relacionada con la historia y el nivel escolar de estos niños, la no participación en programas de habilitación auditiva, la presencia de la deficiencia auditiva desde su nacimiento o en la etapa prelingüística, la ausencia de patologías asociadas tales como problemas visuales no corregidos, discapacidad cognitiva, parálisis cerebral, o bien, trastornos generalizados del desarrollo. Dicha información se utilizó para confirmar las características personales que incluían o excluían a los estudiantes de la muestra.

A partir de lo anterior se seleccionaron tres estudiantes cuyas características eran las siguientes: sordos prelinguales que cursaban el séptimo grado; no habían asistido a terapia, ni usado ayudas auditivas; siempre se habían comunicado en lengua de señas y no tenían patologías asociadas. Los estudiantes fueron contactados en la institución educativa y se corroboró su nivel de comprensión literal en lectura. Para ello, se emplearon estímulos similares a los utilizados posteriormente durante el mapeo cerebral, esto es, Quantitative electroencephalography o Brain electrical activity mapping (qEEG o BEAM).

Considerando su desempeño en esta tarea, se seleccionó finalmente a una estudiante, ya que los 
otros dos candidatos aún no habían desarrollado un nivel de comprensión literal. Las características del sujeto seleccionado se detallan a continuación: joven de 16 años, nacida el 16 de mayo de 1996 en el municipio de Puerto Berrío (Antioquia); inició su escolaridad en ese municipio asistiendo a un aula para sordos, en la que participaban estudiantes de diferentes edades y niveles escolares. Su familia se trasladó a Medellín y allí la estudiante ingresó al tercer grado en una institución enmarcada en los planteamientos de la educación bilingüe bicultural para sordos; por lo tanto, reconoce al sordo como sujeto de derechos, que pertenece a una comunidad lingüística y cuya primera lengua (L1) es la de señas colombiana y la segunda (L2) corresponde al castellano, lengua aprendida en su modalidad lectoescrita $^{21}$. Desde entonces se ha destacado por su buen desempeño académico, que la llevó durante el año 2012 a cursar el séptimo grado. La joven tiene un diagnóstico audiológico de hipoacusia neurosensorial bilateral profunda. La posible causa de la deficiencia auditiva es una enfermedad eruptiva (rubeola) durante el primer trimestre del embarazo. No usa audífonos ni ha participado en programas de habilitación auditiva. Se destaca por ser líder, participa en grupos de teatro y en diferentes actividades dispuestas por la asociación de sordos de la ciudad.

La estudiante asistió en compañía de su acudiente al mapeo cerebral y el consentimiento informado se firmó al inicio del examen. Se la evaluó con la electroencefalografía cuantitativa o mapeo cerebral Quantitative electroencephalography o Brain electrical activity mapping (qEEG o BEAM), prueba no invasiva, la cual registra en un computador la actividad eléctrica cerebral a través de 20 electrodos colocados en el cuero cabelludo ${ }^{7}$, con los cuales se representa dicha actividad cerebral y se la relaciona con áreas anatómicas específicas. Se utilizó la técnica internacional 10-20 en mapas laplacianos, con ondas beta, considerando que dichas ondas aparecen en un individuo despierto en actividad intelectual $^{8}$ y que deben estar en un rango de entre 15 y $30 \mathrm{~Hz}$.

Se utilizó el equipo Aura 24, diseñado para registros de electroencefalograma ambulatorio. EI software Grass Telefactor Twin permitió acceder a los datos y grabar y revisar las capacidades de forma flexible. Teniendo en cuenta estas características, la prueba se realizó en un consultorio.

Una vez que se colocaron los electrodos, se dio a la estudiante la instrucción en lengua de señas acerca de lo que debía hacer durante las tres actividades propuestas.

Las tareas solicitadas se elaboraron considerando la destreza lectora del sujeto estudio del caso, el contexto en el que se presentaban las palabras y las características de las mismas, las cuales estaban relacionadas con la similitud visual, la longitud de la palabra, la regularidad ortográfica, su categoría léxica, la frecuencia, el número de sílabas y su categoría gramatical. Las tres tareas requeridas se describen a continuación.

\section{Tarea 1}

Leer una lista de 20 palabras, es decir, pasar del texto escrito a la lengua de señas. Según Mortón ${ }^{9}$ 
se considera que en la clasificación sintáctica de las palabras existen dos clases de distinciones que parecen relevantes. Por una parte, se distinguen las diferentes categorías gramaticales, mayores $\mathrm{y}$ menores (nombres, verbos, adjetivos, preposiciones, determinantes, etc.); y por otra, se distingue el contraste entre elementos de clase abierta y cerrada (palabras de contenido y función). Esta última distinción está directamente relacionada con los procesos de reconocimiento de palabras, aspectos que se tuvieron en cuenta para la selección de los estímulos. Las palabras seleccionadas para la lectura en esta tarea se ilustran en la Figura 1.

Lee en lengua de señas las siguientes palabras:

1. Sol

2. Gato

3. Elefante

4. Perro

5. Pie

6. Escuela

7. Caballo

8. Amigo

Figura 1. Tarea 1, palabras seleccionadas para la lectura

\section{Tarea 2}

Leer mentalmente oraciones simples, para las que se emplearon 10 imágenes, acompañadas cada una por tres oraciones enunciativas de tipo afirmativo $^{10,11}$ y en donde la participante debía seleccionar aquella que se relacionaba con la lámina. Un ejemplo del tipo de tarea se presenta en la Figura 2.

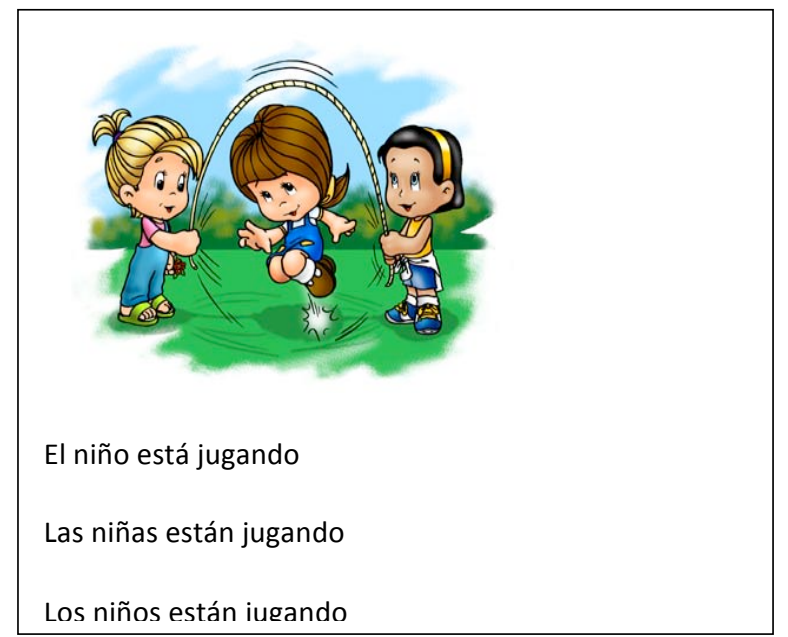

Figura 2: Tarea 2. Ejemplo de imagen acompañada de oraciones nunciativas de tipo afirmativo.

\section{Tarea 3}

Finalmente, la tercera tarea consistió en que la niña leyera un párrafo descriptivo y respondiera a preguntas literales ${ }^{12,13}$ como las que se observan en la Figura 3.

Luis es grande y gordo, siempre está contento; vive en una casa pequeña de color amarillo con ventanas grandes.

1. ¿Dónde vive Luis?

2. ¿Cómo es Luis?

3. ¿Dónde trabaja Luis?

Figura 3: Tarea 3. Ejemplo de párrafo descriptivo con preguntas literales.

Las tareas tuvieron una validación verificada a través de juicio de expertos, proceso en el cual participaron fonoaudiólogas con experiencia en el trabajo con población sorda señante en el escenario escolar. Así mismo, se envió una planilla para evaluar la claridad, pertinencia y suficiencia de las tareas 
descritas. A partir de la retroalimentación recibida, se hicieron ajustes relacionados con el equilibrio en el número de palabras de acuerdo con su extensión, la modificación de una lámina para evitar confusiones y la inclusión de oraciones coordinadas y subordinadas en el párrafo.

El tiempo de registro de la prueba fue de 9 minutos 39 segundos. Para el análisis se utilizó el programa Persyst, con el registro laplaciano, en la modalidad de simetría, que permite realizar un análisis independiente para cada hemisferio cerebral.

\section{Resultados}

A continuación se reportan los resultados globales de la prueba realizada. La información registrada a través del equipo Aura 24 se analizó con el programa Insight, el cual reporta la media y la desviación estándar para el caso particular de las ondas beta, cuyo comportamiento se distribuyó dentro de la curva normal entre 15 y $30 \mathrm{~Hz}$. El registro durante toda la aplicación fue el mismo, es decir, no se presentaron variaciones significativas, con excepción del registro en las áreas correspondientes a la ubicación de los electrodos T3 y T4, en los que las variaciones se encontraron a -3 desviaciones estándar en el hemisferio cerebral izquierdo y a +3 desviaciones estándar en el hemisferio cerebral derecho; lo que permitió evidenciar que las ondas beta del área del lóbulo temporal se encuentran por fuera de la norma. Esta respuesta indica que para el hemisferio izquierdo la onda no alcanza el voltaje mínimo para ser escuchada (por debajo de $15 \mathrm{~Hz}$ ) y que para el hemisferio derecho es muy alta (por encima de $30 \mathrm{~Hz}$ ), por lo que tampoco puede ser escuchada. En conclusión, la respuesta es la misma: no hay funcionalidad en esta área. Un ejemplo de ello es la respuesta representada en la Figura 4, la cual corresponde a la lectura de palabra.

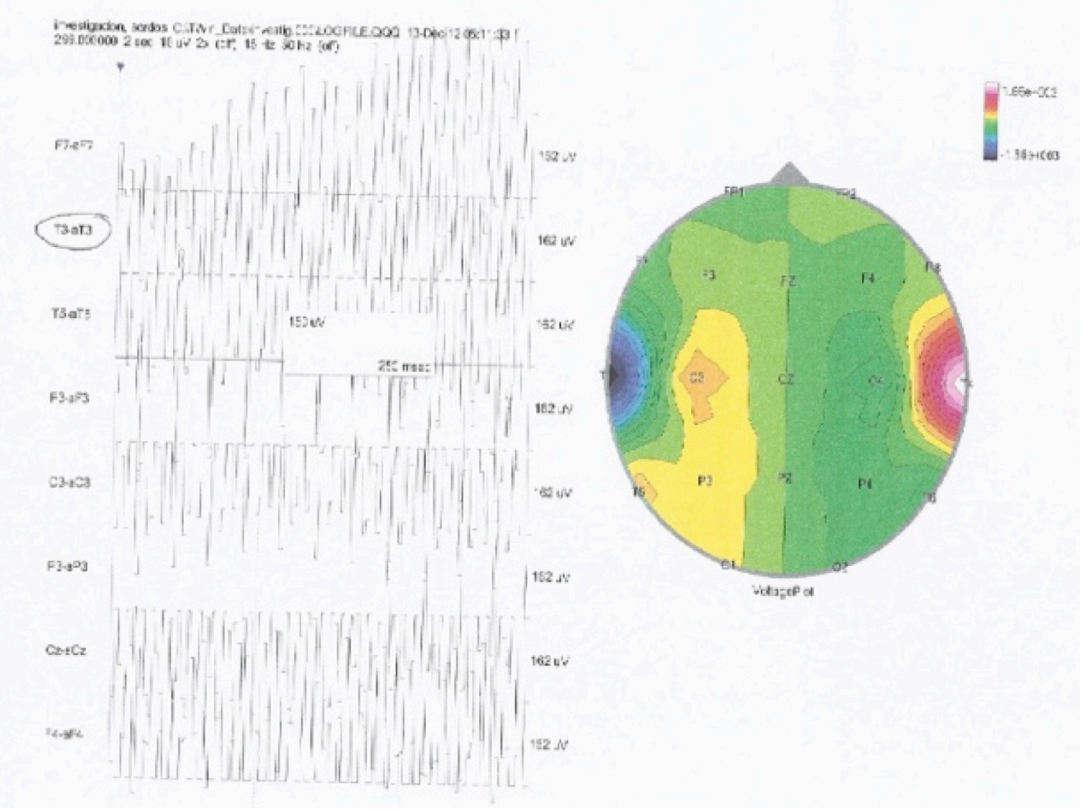

Figura 4: Imagen de mapeo cerebral T3. 
Atendiendo al registro analizado, no existe pues un área de lectura específica a nivel cerebral para el presente caso. El cerebro de la estudiante es funcional para todo tipo de aprendizaje, con excepción del área correspondiente al lóbulo temporal de ambos hemisferios, en la cual se encuentra representada las áreas auditivas primaria circunvolución de Heschl- y secundaria, correspondiente al área 22 y 42 de Brodmann.

\section{Discusión}

Teniendo en cuenta que no existe un referente investigativo con población sorda que permita correlacionar las respuestas registradas, se retomaron las investigaciones desarrolladas con población oyente, reconociendo eso sí la diferencia lingüística.

Afirmar durante el análisis del caso que el desempeño de la estudiante está dentro de la media, con excepción de las áreas correspondientes a la ubicación de los electrodos T3 y T4, permite ratificar las aseveraciones hechas en la investigación de Fiez y Petersen $^{16}$ en la que, basados en modelos neurocognitivos y observando el desempeño en la población oyente, relacionan la lectura de la palabra con la corteza occipital y occipitotemporal. Estos autores coinciden en admitir que tras la recepción de las aferencias visuales en el área visual primaria (bordes de la cisura calcarina en el lóbulo occipital) ocurre una primera identificación de las señales gráficas y su disposición secuencial se da en la corteza secundaria (área 18 y 19 de Brodmann) y desde ahí se reúne la información de ambos hemisferios en la encrucijada temporo-parieto- occipital. En esta área confluyen las aferencias que conducen la información auditiva y visual.

Es importante destacar este aspecto, teniendo en cuenta que los grafemas poseen características fonológicas y espaciales, siendo estas últimas resaltadas por el sordo en el aprendizaje de las representaciones gráficas. Cuetos $^{17}$ precisa la información anterior en población oyente y permite contrastar las respuestas registradas durante la prueba, aclarando que en los procesos léxicos intervienen zonas parieto-temporales en donde se da el reconocimiento de las palabras, las que se agrupan en unidades mayores como frases $u$ oraciones. Dicho análisis sintáctico se realiza en zonas perisilvianas y, por último, la integración del mensaje de la oración con sus propios conocimientos análisis semántico- ocurre fundamentalmente en los lóbulos frontales.

De acuerdo con los datos obtenidos por el mapeo en la zona que coincide con la corteza auditiva, se evidenció que las ondas T3 se ubicaron en -3 desviaciones estándar en el hemisferio izquierdo, situándose en el límite inferior de la curva ( $5 \%$ inferior de la curva normal o campana de Gauss) y en T4 a +3 desviaciones estándar en el hemisferio derecho, quedando ubicada en el límite superior de la curva (5\% superior en la curva normal); información que permite afirmar que no hay funcionalidad en estas zonas. Por lo tanto, al no existir huellas mnémicas con información fonológica, no se establece una relación grafema-fonema, es decir, no se evidencia una transformación de la palabra escrita en la representación fonológica del sonido de la misma. Este aspecto caracteriza la muy 
ampliamente descrita ruta fonológica o indirecta, contemplada dentro del modelo de doble ruta en la lectura $^{22}$ y en donde la recodificación fonológica se realiza de forma obligatoria y por medio de un sistema de reglas de conversión grafémico-fonémica que es considerado como vía para acceder al léxico mental, según Rubistein et al. (1971) citado por Belinchon et al. ${ }^{15}$. Por lo tanto, para el caso en particular, se reconocería la ruta semántica o directa como la forma de procesar la información escrita.

Vale destacar que no existen investigaciones que muestren cuál es la incidencia de dicha ruta en el aprendizaje de la lectura en una población sorda señante. Ahora bien, existen múltiples factores que influyen en este aprendizaje. En efecto, autores como Reynvoet $^{23}$, Cuetos $^{24}$ y Reynoso $^{22}$ reconocen el importante papel que juega la conversión grafemafonema para los oyentes en las etapas iniciales de desarrollo de la lectura, llegando a relacionar una dificultad en la ruta grafo-fónica con la imposibilidad para leer. Sin embargo, reconocen igualmente que el grado de participación de esta ruta disminuye con el avance de la lectura.

Es interesante observar el cambio en la lateralización que ocurre en las modalidades visual y auditiva, lo cual sugiere que los mecanismos del cerebro que apoyan la integración semántica de los estímulos significativos funcionan de manera independiente, siendo el sistema semántico común para todas las modalidades perceptuales. Newman ${ }^{18}$ afirma que los cambios en la activación del plano temporal no son idénticos en cada uno de los hemisferios al momento de recibir la información visual y auditiva, por lo que sugiere que el papel de dichos planos (cerebro izquierdo y derecho) depende de la modalidad de la lengua usada.

Por lo tanto, de acuerdo con los planteamiento de Tirapu-Ustárroza, J., Muñoz, J.M. y Pelegrín, C. ${ }^{19}$, en el caso de estudio no existe un almacén fonológico asistido por un proceso de control basado en el repaso articulatorio, que actúe como sistema de almacenamiento y que permita utilizar un lenguaje subvocal, función que incluye el bucle fonológico, descrito por el autor. Dicho componente de la memoria de trabajo permite el almacenamiento transitorio del material verbal implicado en las tareas de memoria a corto plazo. No existen, entonces, huellas de memoria relacionadas con dicha información fonológica. Estos datos se han remplazado por una información viso espacial que se alimenta directamente de la percepción visual, o bien, indirectamente a partir de la generación de imágenes visuales, siendo estas empleadas para crear huellas mnemotécnicas que apoyan la relación de la palabra escrita con un archivo de significados construido a partir de una lengua ágrafa de carácter viso espacial. Esta información permite ratificar la importancia de fortalecer la memoria de trabajo a partir de estímulos presentados a través del canal visual.

En relación con la dinámica del cerebro, la respuesta a la pregunta acerca de dónde se archiva esta información se puede interpretar a la luz de las afirmaciones hechas por Kolb, B. y Whishaw, I. ${ }^{14}$, quienes reconocen que dentro de las funciones del lóbulo temporal está el reconocimiento de los objetos, la selección, organización y categorización de 
los estímulos visuales, la memoria del neocórtex y la

capacidad para utilizar la información contextual.

Los resultados de la presente investigación deben ser enriquecidos con trabajos realizados con la población objeto de estudio, apoyados en técnicas como la resonancia magnética funcional, con el objetivo de precisar la descripción del funcionamiento del cerebro durante el proceso lector. Así mismo, es importante replicar esta experiencia investigativa en otros grupos de investigación, cuyo interés esté centrado en apoyar el proceso de aprendizaje o enseñanza de la lectura y escritura como segunda lengua y como modalidad de comunicación empleada en interacciones comunicativas efectivas de la población sorda.

\section{Agradecimientos}

Se destacan los aportes de la doctora Clara Inés King de Larrarte, quien realizó la electroencefalografía cuantitativa o mapeo cerebral y del doctor Juan Diego Betancur Arias, quien con sus aportes enriqueció el análisis de la investigación.

\section{Referencias}

1. Rojas, A. (2005). Representaciones sociales de un grupo de estudiantes sordos frente a la lectoescritura. Revista ARETE, 4 y $5,12-17$.

2. Skliar, C. (2013). La educación bilingüe para los sordos. http://www.espaciologopedico.com/articulos/articulos 2.php?Id_articulo=306. Visita 07-03-2013.

3. Oviedo, A. (2001). Apuntes para una gramática de la lengua de señas colombiana. Cali: Universidad del Valle/INSOR.

4. Flórez, R. (2004). El lenguaje en la educación. Una perspectiva fonoaudiológica. Bogotá: Universidad Nacional de Colombia.

5. Sacks. O. (1999), Veo una voz. Salamanca: Anaya \& Mario Muchnik,.

6. Ortiz, L., Álvarez, L. y González, R. (2008). Modelos anatomotopográficos de las áreas cerebrales que se activan durante la función lingüística. Revista de neurología, Vol. 47, 12, 653-658.

7. Matusevich, D., Ruiz, M. y Vairo, M. (2002). EEG cuantitativo y mapa cerebral. Evolución histórica, aplicaciones clínicas y controversias epistemológicas. Revista argentina de psiquiatría VERTEX, Vol. XIII, 48, 198-204.

8. García, H. y Quian Quiroga, R. (1999). La utilidad de los métodos de análisis cuantificado del EEG en neurofisiología clínica. Arch Neurol Neurocir Neuropsiq., Vol 2, 34-43.

9. Morton, J. (1990). Reconocimiento de palabras. En: Valle, F. y Cuetos, F. Lecturas de psicolingüística. Comprensión y producción del lenguaje. Madrid: Alianza Editorial, pp. 99-138.

10. Rodríguez, T. (2008). Estudio sintáctico y discursivo de algunas estructuras enunciativas y citativas del español. http://dialnet.unirioja.es/servlet/articulo?codigo=2926 056. Visita 06-05-2013.

11. González, J. (2001). Revisión de la clasificación de la oración según el MODUS. Anuario de estudios filosóficos, XXIV, 207-221. 
12. Dubois, M. (1984). Algunos interrogantes sobre la comprensión de la lectura: Lectura y Vida. http://www.lecturayvida.fahce.unlp.edu.ar/numeros/a 5n4/05_04_Dubois.pdf. Visita 11-09-2012.

13. Quintana, H. (2004). La enseñanza de la comprensión lectora. Espacio logopédico.com. http://www.espaciologopedico.com/articulos/articulos 2.php?ld_articulo=498. Visita 11-09-2012.

14. Kolb, B. y Whishaw, I. (2006). Neuropsicopatología humana. 5a edición. Madrid: Editorial Médica Panamericana,

15. Belinchon, M., Riviere, A. y Igoa, J. (1992). Psicología del lenguaje: Investigación y teoría. Madrid: Editorial Trotta, pp. 363-412.

16. Fiez, J. and Petersen, S. (1998). Neuroimaging studies of Word Reading. Proc Natl Acad Sci USA, Vol. 95, 914921.

17. Cuetos, F. (2011). Psicología de la Lectura. 8a edición. Barcelona: Wolters Kluwer Educación.

18. Newman-Norlund, R.D., Frey, S.H., Petitto, L.A. and Grafton, S.T. (2006). Anatomical substrates of visual and auditory miniature Second-Language Learning. Journal Cognitive Neuroscience. http://www.ncbi.nlm.nih.gov/pubmed/17129186.

Visita 14-09-2013.

19. Tirapu-Ustárroza, J., Muñoz, J.M. y Pelegrín, C. (2002). Funciones ejecutivas: Necesidades de una integración conceptual. En Revista de Neurología 34, 7, 673-685. http://www.neurologia.com/pdf/Web/3407/m070673. pdf. Visita 17-04-2013.

20. Ministerio de Educación Nacional. Instituto Nacional para Sordos, INSOR. Ramírez, P. y Castañeda, M. (2003). Educación bilingüe para sordos. Generalidades. http://www.colombiaaprende.edu.co/html/mediateca/ 1607/articles-88150_archivo.pdf. Visita 26-02-2013.

21. Ministerio de Educación Nacional. Instituto Nacional para Sordos, INSOR. (2006). Educación bilingüe para sordos, Etapa escolar, Orientaciones pedagógicas. http://portal.insor.gov.co/images/PUBLICACIONES/cart illa_etapa_escolar.pdf Visita 25-02-2013.

22. Reinoso, V., Bernal, J., Silva Pereyra, J., Rodríguez, M., Yáñez, G., Fernández, T. y Del Río, Y. (2010).
Procesamiento fonológico y léxico en niños normolectores de educación primaria. Infancia y aprendizaje, Vol. 33, 3, 413-425. http://www.ingentaconnect.com/content/fias/iya/201 0/00000033/00000003/art00010. Visita 14-08-2013.

23. Reynvoet, B., Brysbaert, M. y Fias, W. (2002). Priming semántico en el número de nombres. The Quarterly Journal of Experimental Psychology, Vol. 55, 4, 11271139.

24. Cuetos, F. (2002). Sistemas de lectura en ortografías transparentes: evolución de la dislexia profunda en español. Fundación Infancia y Aprendizaje, Vol. 14, 2, 133-149.

http://www.ingentaconnect.com/content/fias/cog/200 2/00000014/00000002/art00001. Visita 14-08-2013.

25. Perani, D., Paulesu, E., Galles, N.S, Dupoux, E., Dehaene, S., Bettinardi, V., Cappa, S.F., Fazio, F. and Mehler, J. (1998). The bilingual brain proficiency and age of the second language. http://brain.oxfordjournals.org/content/121/10/1841.f ull.pdf. Visita 21-09-2012.

26. Neville, H., Bavelier, D., Corina, D., Rauschecker, J., Karni, A., Lalwani, A., Braun, A., Clark, V., Jezzard, P. and Turner, R. (1998). Cerebral organization for language in deaf and hearing subjects: Biological constraints and effects of experience. http://www.pnas.org/content/95/3/922.full.pdf. Visita 14-09-2012.

27. Figueroa, V. y Lissi, M. (2005). La lectura en personas sordas: consideraciones sobre el rol del procesamiento fonológico y la utilización del lenguaje de señas. Estudios pedagógicos XXXI, No 2, 105-119. Chile. http://www.scielo.cl/scielo.php?pid=S071807052005000200007\&script=sci_arttext. Visita 10-092012. 